\title{
Automatic Detection and Removal of Fiducial Markers Embedded in Fluoroscopy Images for Online Calibration
}

\author{
Laurence Smith, Mike Pleasance, Rosalyn Seeton, Neculai Archip, and \\ Robert Rohling \\ University of British Columbia, Canada. \\ Software: http://www.ece.ubc.ca/ neculaia/Matlab_code_fluoroscopy.htm
}

\section{Introduction}

Computer assisted fluoroscopic navigation has received strong interest as a new tool for various medical interventions (e.g. spinal, orthopaedic and brachytherapy procedures). One of the challenges for the intraoperative use of c-arm imaging is on-line calibration 12 . Usually the method selected to perform this task involves placing a grid of fiducial markers in the x-ray path. The real geometry of the grid is known, so if the geometry of the grid in the image can be found then the real location of the other features in the image can be determined. Detection of the markers is the most important step in the calibration process [2]. This paper describes a new technique for automatic detection of markers. The purpose is to detect the highest number of markers as possible from a standard grid. As a convenience, removal of the detected markers is also proposed.

\section{Methodology}

Finding fiducial markers with an automatic algorithm is difficult because of the presence of tools and dark bones that obscure the markers, variability of the marker appearance and the arbitrary orientation of the grid. To allow the algorithm to work on a variety of c-arm scanners, the only assumptions are the approximate size and shape of the markers (small crosses between 5 and 20 pixels wide) and that they are regularly spaced. Given these assumptions, the following steps are performed. First, the outer portion of the image is masked using a circular Hough transform. This involves a search for two symmetric maximum to accommodate the slightly elliptical shape of the field-of-view from most carm scanners. Next, the image is convolved with a marker-shaped kernel and subtracted from the original image to emphasize the markers. Groups of pixels that exhibit full or partial marker shapes are flagged. The candidates are then pruned by rejecting flagged locations that do not fall at regular locations. The remaining candidates are assigned to bands by using a process similar to raster scanning. At this stage, the highly visible markers have been identified, and their regularly spaced locations can be used to recover weaker markers. So a virtual grid is constructed by fitting curves to the markers in each band and the virtual 
grid intersections are used to recover the weaker markers. The complete list of markers is then used for on-line calibration. As an optional step, the markers are removed by replacing marker pixels with values obtained by spline-interpolation of nearby pixels. An example of marker detection and removal is given in Fig. 1.
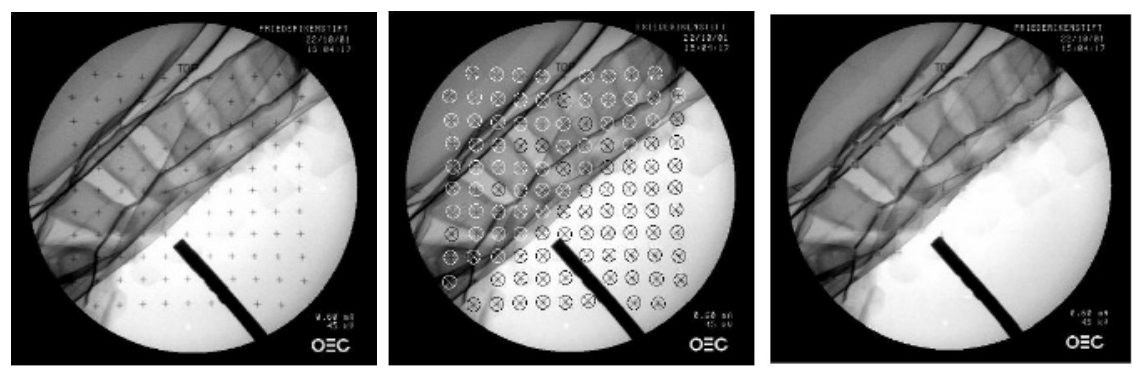

Fig. 1. The majority of the markers are located despite the presence of bones, tools and other obstructing features. The original image, circles around the detected markers, and the image after marker removal are shown.

\section{Results and Conclusion}

The algorithm was tested on 97 clinical images $(768 \times 576$ pixels $)$. The images are from both brachytherapy and orthopaedic surgeries. Various c-arm scanners were used including a Siemens Siremobil ISO C (Siemens Medical Solutions, Erlagen, Germany), GE/OEC Compact 7700 (General Electric, Milwaukee, WI) and Toshiba STX 650A (Toshiba America Medical Solutions, Tustin, CA). Of the 12066 possible fiducial markers, 11718 were successfully found, for a success rate of $96.28 \%$. The success rate for each image is defined as the number of correctly located markers to the number of correctly located, extra and missing markers, so that $100 \%$ is only achieved if there are no extra or missing markers. Of the 97 images, only 16 had less than 95\%. The best case identified 100\% and the worst case identified $76 \%$. This detection rate is comparable to manual marker identification and comparable to the number deemed sufficient for other grids [2]. Compared to manual identification, tests show the markers were located to within $1.30 \pm 0.89$ pixels, which corresponds to $0.47 \pm 0.32 \mathrm{~mm}$. Overall, the algorithm is suitable for clinical applications.

\section{References}

1. P. Tate, V. Lachine, L. Fu, H. Croitoru, M. Sati, Performance and Robustness of Automatic Fluoroscopic Image Calibration in a New Computer Assisted Surgery System, MICCAI 2001, LNCS 2208, pp. 1130-1136, 2001.

2. H. Livyatan, Z. Yaniv, L. Joskowicz, Robust automatic C-arm calibration for fluoroscopy-based navigation: a practical approach, MICCAI 2002, LNCS 2488, pp. 60-68, 2002. 